\title{
Transport of Photoexcited Electron-Hole Plasma in GaN/AlGaN Quantum Well
}

\author{
K.P. Korona ${ }^{a}$, P. CABAn $^{b}$ And W. Strupiński ${ }^{b}$ \\ ${ }^{a}$ Institute of Experimental Physics, University of Warsaw, Hoża 69, 00-681 Warsaw, Poland \\ ${ }^{b}$ Institute of Electronic Materials Technology, Wólczyńska 133, 01-919 Warsaw, Poland
}

\begin{abstract}
We report spatially resolved photocurrent measurements showing transport of excitation on long distances in plane of a $6 \mathrm{~nm} \mathrm{GaN} / \mathrm{Al}_{0.1} \mathrm{Ga}_{0.9} \mathrm{~N}$ quantum well. The strong field present in nitrides (due to large spontaneous and piezoelectric polarizations) leads to lower recombination rates of electrons and holes, so in the case of electron-hole pairs excited by light, relatively long-lived electron-hole plasma could be generated. In the case of the investigated quantum well, lifetime of few $\mu$ s was expected. The thermal measurements showed that barriers were low enough, so all excited carriers could reach the electrode (thermal activation energy of $0.11 \mathrm{eV}$ was found). The diffusion length for unbiased structure was about $40 \mu \mathrm{m}$. It was observed that the charge transport could be clearly accelerated by bias. In the biased quantum well, the transport range was of the order of $100 \mu \mathrm{m}$ under both positive and negative bias. The reported effect of long transport range is very important for electronic devices made on the $\mathrm{GaN} / \mathrm{AlGaN}$ structures.
\end{abstract}

PACS numbers: 73.61.Ey, 72.40.+w

\section{Introduction}

Nitride structures are used for LEDs, high electron mobility transistors and UV detectors [1], including detectors with spectral response tunable by external voltage [2]. In all these devices, as well as in other nitride structures, high electric field generated by spontaneous and piezoelectric polarization is present [3]. The strong field leads to separation of electrons and holes in these structures $[4,5]$ and reduction of the electron and hole functions overlap. So the recombination rate decreases and long-lived electron-hole plasma can be generated. The carriers lifetime and transport range along the quantum well channel is important for field effect transistors with wide gates like phototransistors and chemical sensors.

\section{Experimental}

The reported GaN/AlGaN structure was grown by metal organic chemical vapor deposition (MOCVD) on sapphire substrate. A $6 \mathrm{~nm}$ wide, undoped GaN QW inserted between undoped $\mathrm{Al}_{0.1} \mathrm{Ga}_{0.9} \mathrm{~N}$ layers was grown on a conductive $\mathrm{Al}_{0.1} \mathrm{Ga}_{0.9} \mathrm{~N}$ layer. Semitransparent Schottky contact forming a gate (and collecting electrode) was made by evaporation of gold. The structure was investigated in the temperature range from 4 to $300 \mathrm{~K}$ by photoluminescence (PL) and spatially-resolved photocurrent (PC) measurements. The light spot was formed in a $30 \mu \mathrm{m}$ wide line and the PC spectra were measured as a function of distance between the light spot and the electrode.

\section{Results and discussion}

At room temperature photocurrent spectra showed two thresholds at 3.35(5) eV and at 3.68(5) eV connected with the GaN QW and the $\mathrm{Al}_{0.1} \mathrm{Ga}_{0.9} \mathrm{~N}$ barrier, respectively (see Fig. 1). The threshold energies increased monotonically with decrease of temperature. At $4 \mathrm{~K}$, the QW and AlGaN PC had thresholds at 3.48(5) eV and 3.75(5) eV, respectively. The PL signal was very weak which suggested that radiative recombination rate was very low (and radiative recombination time was very long). The energy of the PL emission of the QW was at $3.21 \mathrm{eV}$ which gave strong Stark shift $(\Delta E=0.27(6) \mathrm{eV})-$ due to the high electric field in the structure.

Amplitude of the PC signal from the AlGaN barrier was nearly independent of temperature. On the other hand, signal from the QW increased strongly with increasing temperature (see inset in Fig. 1). The effect can be explained by assumption that the photocurrent was limited by transport from the GaN QW to the AlGaN barriers. The carriers could go out off the QW by thermally activated jumps. The thermal activation energy was found $E_{\mathrm{A}}=0.11(1) \mathrm{eV}$, which is similar to the value expected for the valence-band offset [6]. At temperature of about $250 \mathrm{~K}$ the current saturated which means that all excited carriers took part in photocurrent.

Photocurrent spectra measured at various biases showed the change of sign of the photocurrent. At zero or reverse bias the whole photocurrent spectrum was in reverse direction. At low forward bias $\left(U_{\mathrm{F}}<1 \mathrm{~V}\right)$ the $\mathrm{PC}$ of the barrier was still in reverse direction while the current excited in the QW was forward. The direction 


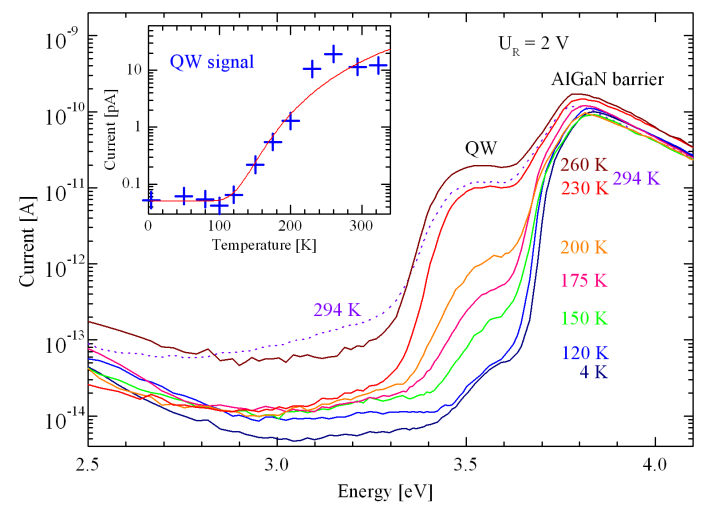

Fig. 1. PC spectra vs. T (light focused on the electrode). The signal from the QW changes significantly. Inset: the QW signal fitted with theoretical curve.

of the current revealed electric field distribution in the structure. Due to spontaneous polarization, the electric field in the unbiased structure was negative (in reverse direction). The forward external bias added positive component to the field, so direction of the field in the QW changed.

In spatially-resolved photocurrent measurements, carriers were excited at distance $x$ from the Schottky-type electrode. The results were plotted in Fig. 2. It can be noticed that the PC signal from the barriers decreased fast with the distance while the QW signal persisted longer. For example, at $x=200 \mu \mathrm{m}$ signal from the barriers was 2 orders of magnitude weaker than at $x=0$, while photocurrent from the QW decreased only 6 times. It is worth to notice that this result was obtained under bias (both forward and reverse). At no bias case, the signal from the QW dropped 2 orders of magnitude, similarly like the signal from the barrier.

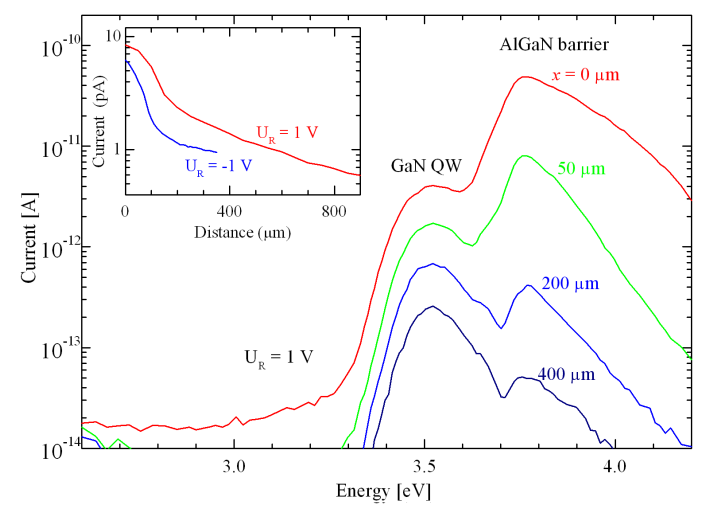

Fig. 2. PC spectra excited at various distances from the electrode, $x$, for reverse bias $U_{\mathrm{R}}=1 \mathrm{~V}$, at $295 \mathrm{~K}$. Inset: the QW photocurrent vs. distance.

Taking into account that concentration of electronhole pairs (EHPs) is $n_{\mathrm{eh}}(x, y)$, the EHP flux $\phi(x, y)$ can be calculated as

$$
\begin{aligned}
& \phi(x, y)=-D\left[n_{\mathrm{eh}}(x, y) \nabla E_{\mathrm{p}}(x, y) / k_{\mathrm{B}} T\right. \\
& \left.-\nabla n_{\mathrm{eh}}(x, y)\right]
\end{aligned}
$$

where $D$ is diffusion constant and $E_{\mathrm{p}}(x, y)$ is potential energy of the EHP in the electric field perpendicular to the QW plane, $F_{z}(x, y)$. The EHP energy in the field is lower which was observed as a Stark shift in luminescence measurements $[4,5]$. The dependence of the $E_{\mathrm{p}}$ vs. $F_{z}$ is generally nonlinear, but for QW of width $d$, it can be approximated as $E_{\mathrm{p}}=-\left|e d F_{z}\right|$, so it is always negative and the EHP flux is directed by the gradient of $\left|F_{z}(x, y)\right|$.

In the case of zero bias, the $F_{z}(x, y)$ and the $E_{\mathrm{p}}(x, y)$ are constant and we observe only diffusion of the carriers. In this case, the decrease of the signal was described by equation $I(x)=I_{0} \exp \left(-x / L_{\mathrm{D}}\right)$. The diffusion length was found $L_{\mathrm{D}}=40 \pm 30 \mu \mathrm{m}$. This value is reasonable for electrons of few $\mu$ s lifetime, expected for such QW [4].

When in spatially-resolved PC experiment we changed the external bias, we observed that the carriers were attracted from longer distances. Both positive and negative bias attracted carriers to the gate which was consistent with the conclusion from Eq. (1) that the EHPs were attracted to the stronger electric field and the direction of the field is not important.

In the case of the biased QW, the shape of the $\mathrm{PC}(x)$ dependence was similar to Gaussian curve (see inset in Fig. 2). The widths of the Gaussian curves fitted to the data were 100 and $120 \mu \mathrm{m}$ for forward and negative bias, respectively $\left(\left|U_{\mathrm{B}}\right|=1 \mathrm{~V}\right)$. For higher biases the currents were higher but the widths (range of transport) were similar.

The time-resolved photocurrent decays were measured with excitation at various distances from the electrode. The PC decays were slow and seemed to be two-exponential. The slower component $\left(\tau_{\mathrm{S}}=570 \mathrm{~ms}\right)$ was similar for all distances. It can be connected with trapping of the carriers on some defects and a retarded escape. The faster component was $\tau_{\mathrm{F}}=90 \mathrm{~ms}$ at the electrode $(x=0)$ and $\tau_{\mathrm{F}}=120 \mathrm{~ms}$ for excitation at $x=200 \mu \mathrm{m}$. So the $\tau_{\mathrm{F}}$ time was characteristic for the moving carriers, however this was much longer than the expected lifetime of the carriers.

\section{Conclusion}

We conclude that the carriers excited in GaN/AlGaN quantum well can flow in a range of about hundred micrometers. This fact can be explained by high diffusion constant and long lifetime of electron-hole pairs in the QW. The transport in the QW has long range for both positive and negative bias. This result is consistent with the fact that electron-hole pairs are attracted to the electric field of higher absolute value.

\section{Acknowledgments}

We would like to acknowledge that this work was partially supported by EU project No. MTKD-CT-2005-029671. 


\section{References}

[1] W. Yang, T. Nohava, S. Krishnankutty, R. Torreano, S. McPherson, H. Marsh, Appl. Phys. Lett. 73, 1086 (1998).

[2] K.P. Korona, A. Drabińska, P. Caban, W. Strupiński, J. Appl. Phys. 105, 083712 (2009).

[3] F. Bernardini, V. Fiorentini, D. Vanderbilt, Phys. Rev. B 56, R10024 (1997).
[4] J.S. Im, H. Kollmer, J. Off, A. Sohmer, F. Scholz, A. Hangleiter, Phys. Rev. B 57, R9435 (1998).

[5] K.P. Korona, J. Borysiuk, C. Skierbiszewski, S. Porowski, Acta Phys. Pol. A 112, 243 (2007).

[6] G. Martin, A. Botchkarev, A. Rockett, H. Morkoc, Appl. Phys. Lett. 68, 2541 (1996). 